OPEN ACCESS

Edited by:

Teng Ma,

Capital Medical University, China

Reviewed by:

Magdalena Boguta,

Institute of Biochemistry

and Biophysics (PAN), Poland

Mehdi Kabani,

Centre National de la Recherche

Scientifique (CNRS), France

*Correspondence:

Francisco Navarro

fngomez@ujaen.es

Specialty section:

This article was submitted to

Protein and RNA Networks,

a section of the journal

Frontiers in Molecular Biosciences

Received: 18 February 2021

Accepted: 31 March 2021

Published: 28 April 2021

Citation:

Garrido-Godino Al,

Gutiérrez-Santiago F and Navarro F (2021) Biogenesis of RNA

Polymerases in Yeast.

Front. Mol. Biosci. 8:669300.

doi: 10.3389/fmolb.2021.669300

\section{Biogenesis of RNA Polymerases in Yeast}

\author{
Ana I. Garrido-Godino ${ }^{1}$, Francisco Gutiérrez-Santiago ${ }^{1}$ and Francisco Navarro ${ }^{1,2 *}$ \\ ${ }^{1}$ Departamento de Biología Experimental-Genética, Universidad de Jaén, Jaén, Spain, ${ }^{2}$ Centro de Estudios Avanzados en \\ Aceite de Oliva y Olivar, Universidad de Jaén, Jaén, Spain
}

Eukaryotic RNA polymerases (RNA pols) transcriptional processes have been extensively investigated, and the structural analysis of eukaryotic RNA pols has been explored. However, the global assembly and biogenesis of these heteromultimeric complexes have been narrowly studied. Despite nuclear transcription being carried out by three RNA polymerases in eukaryotes (five in plants) with specificity in the synthesis of different RNA types, the biogenesis process has been proposed to be similar, at least for RNA pol II, to that of bacteria, which contains only one RNA pol. The formation of three different interacting subassembly complexes to conform the complete enzyme in the cytoplasm, prior to its nuclear import, has been assumed. In Saccharomyces cerevisiae, recent studies have examined in depth the biogenesis of RNA polymerases by characterizing some elements involved in the assembly of these multisubunit complexes, some of which are conserved in humans. This study reviews the latest studies governing the mechanisms and proteins described as being involved in the biogenesis of RNA polymerases in yeast.

Keywords: RNA polymerases, biogenesis, assembly, transcription, yeast

\section{INTRODUCTION}

Transcription is the most studied step of the gene expression catalyzed by RNA polymerases (RNA pols). Eukaryotes contain at least three RNA pols (RNA pols I, II, III), while archaea and bacteria consist of a single enzyme (Werner and Grohmann, 2011). In addition, two additional RNA pols have been described to be present in plants (RNA pols IV and V) (Wierzbicki et al., 2008; Haag and Pikaard, 2011; Haag et al., 2014). Although single-subunit RNA pols exist (as in bacteriophage T7) (Kwapisz et al., 2008), the bacterial, archaeal, and eukaryotic RNA pols are heteromultimeric complexes (Werner and Grohmann, 2011; Cramer, 2019). In eukaryotes, RNA pol I comprises 14 subunits and synthesizes a precursor of the three largest rRNAs (Werner et al., 2009; Lane et al., 2011; Werner and Grohmann, 2011; Moreno-Morcillo et al., 2014). RNA pol II contains 12 subunits and is responsible for the transcription of mRNAs and some non-coding RNAs (Armache et al., 2003, 2005; Werner and Grohmann, 2011). RNA pol III is composed of 17 subunits and catalyzes the synthesis of tRNAs and 5S rRNA, as well as other non-coding RNAs (Werner et al., 2009; Fernández-Tornero et al., 2011; Lane et al., 2011; Werner and Grohmann, 2011; Dieci et al., 2012; Khatter et al., 2017). 
Despite the fact that the transcription process and regulation have been extensively studied along with the structure of RNA pols (Ishihama, 1981; Briand et al., 2001; Cramer, 2002, 2019; Armache et al., 2003, 2005; Werner, 2007; Werner et al., 2009; Fernández-Tornero et al., 2011, 2013; Lane et al., 2011; Werner and Grohmann, 2011; Moreno-Morcillo et al., 2014), very little is known about the biogenesis of multisubunit RNA pols and how these processes occur in yeast. Several studies have identified many factors involved in the assembly and/or nuclear transport of RNA polymerases in both yeast and human cells, with most of them operating for the biogenesis of RNA pol II. In light of this, this review focuses on pre-existing knowledge of the assembly of RNA polymerases in yeast.

\section{GENERAL OVERVIEW OF THE ASSEMBLY PROCESSES OF RNA POLYMERASES}

A model for bacterial RNA pol ( $\alpha \alpha \beta \beta^{\prime} \omega$ subunits) assembly has been proposed based on in vitro experiments. Assembly would start with the formation of the $\alpha \alpha$ dimer, which would interact with the $\beta$ subunit. Later, the $\alpha \alpha \beta$ module would associate with the $\beta$ ' subunit, which probably forms a complex with the $\omega$ subunit (Ishihama, 1981). Interestingly, the $\omega$ subunit, which is not essential, seems to stabilize the $\beta$ ' subunit (Minakhin et al., 2001).

In yeast, a model for the biogenesis of RNA pol II based on bacterial RNA pol formation (Ishihama, 1981) has been suggested (Wild and Cramer, 2012). A similar model has been proposed in human cells, which suggests the conservation of these processes. Yeast RNA pol II possesses the bacterial homolog $\alpha \alpha \beta \beta^{\prime} \omega$ core composed of subunits Rpb1, Rpb2, Rpb3, Rpb11, and Rpb6 (Zhang et al., 1999; Werner and Grohmann, 2011; Wild and Cramer, 2012; Cramer, 2019). These subunits are conserved in yeast RNA pol I and III (Werner and Grohmann, 2011; Cramer, 2019). Accordingly, the Rpb3 subassembly complex (corresponding to $\alpha \alpha$ dimer: Rpb3, Rpb10, Rpb11, and Rpb12) would form and interact with the Rpb2 subassembly complex (similar $\beta$ subunit: Rpb2 and Rpb9) prior to the association with the Rpb1 subassembly module ( $\beta^{\prime} \omega$ subunits: composed of Rpb1, Rpb5, Rpb6, and Rpb8). Rpb6 ( $\omega$, subunit) would act by stabilizing the largest subunit of RNA pol II for the assembly of RNA pol II (Nouraini et al., 1996; Minakhin et al., 2001; GarridoGodino et al., 2013) in line with the role proposed for the $\omega$ subunit (Minakhin et al., 2001). Finally, the stalk subcomplex (Rpb4/7) would associate with the preassembled core enzyme (Wild and Cramer, 2012 and our unpublished data), although it can dissociate (Armache et al., 2005).

While the proposed model accounts for RNA pol II, the question of what occurs for RNA pol I and III arises. By taking into account the conservation of the different subunits $\alpha \alpha \beta \beta^{\prime} \omega$ in RNA pol I and III, similar pathways may act for the assembly of all eukaryotic RNA pols, and this process is likely coordinated by the existence of five RNA pols common subunits: Rpb5, Rpb6, Rpb8, Rpb10, and Rpb12 (Wild and Cramer, 2012). It is worth noting that the yeast homologous $\omega$ subunit Rpb6 has also been described to stabilize RNA pol I in addition to RNA pol II (Nouraini et al., 1996; Minakhin et al., 2001; GarridoGodino et al., 2013). Furthermore, the availability of the shared subunit Rpb12 must be a limiting step in the assembly of the three RNA pols in yeast (Rubbi et al., 1999; Wild and Cramer, 2012). In addition, homologous Rpb4/7 complexes exist for RNA pol I and III (Rpa14/Rpa43 and Rpc17/Rpc25, respectively) (Werner and Grohmann, 2011). Nevertheless, the mechanisms governing the assembly of RNA pol I and III are described in another article published in the same issue by Boguta and Turowski (in press).

In line with previously proposed mechanisms for yeast, quantitative proteomic analyses in human cells have demonstrated the existence of a cytoplasmic RNA pol II subcomplex formed by subunits RPB2, RPB3, RPB10, RPB11, and RPB12 (Boulon et al., 2010), which suggests that the interaction of the RPB2 and RPB3 subassembly complexes may occur prior to the association with the RPB1 subassembly complex.

Another important question relates to where the assembly of the RNA polymerases occurs and how they enter the nucleus. RNA pol II assembly has been proposed to occur in the cytoplasm before its nuclear import in yeast (Boulon et al., 2010; Corden, 2011; Wild and Cramer, 2012; Mirón-García et al., 2013; Gómez-Navarro and Estruch, 2015), as it similarly occurs in human cells (Boulon et al., 2010; Corden, 2011; Wild and Cramer, 2012; Mirón-García et al., 2013; GómezNavarro and Estruch, 2015), and as it is suggestes by both the cytoplasmic accumulation of RNA pol II subunits after blocking biogenesis and by the identification of RNA pol II transport factors (i.e., Iwr1 and Rtp1) suggest (Czeko et al., 2011; Gómez-Navarro et al., 2013). However, additional Iwr1independent mechanisms have been proposed to allow some RNA pol II subunits to passively diffuse into the nucleus in both yeast and human (Boulon et al., 2010; Gómez-Navarro and Estruch, 2015). Notably, cytoplasmic biogenesis has also been proposed for yeast RNA pol I and III based on the cytoplasmic accumulation of the largest subunits of their RNA pols under impairing assembly (Mirón-García et al., 2013). Although the mechanisms governing the assembly of RNA pol I and III are not as clear, mass spectrometry approaches and dissociation studies of the elongation complexes in yeast have identified the disassembly of RNA pol I and III and probably assembly subcomplexes (Schneider and Nomura, 2004; Lane et al., 2011). In yeast, the RNA pol III core may be assembled in the cytoplasm (Hardeland and Hurt, 2006; MirónGarcía et al., 2013), whereas additional subcomplexes or free subunits must bind the core in the nucleus (Hardeland and Hurt, 2006). In fact, there are reports informing that the cytoplasmic accumulation of the second largest subunit Rpc128 also leads to the accumulation of other subunits, such as Rpc160, Rpc53, and Rpc11, whereas others remain nuclear (Hardeland and Hurt, 2006). Interestingly, dissociation analyses by mass spectrometry have evidenced that several subcomplexes appear after disturbing the RNA pol III structure in vitro, including stable trimer Rpc31/82/34 and the two heterodimers Rpc82/31 and Rpc17/25, as well as some free subunits like Rpb10, Rpc11, Rpc82, and Rpc34 (Lane et al., 2011). Notably, 
human RNA pol I assembly has been proposed to be sequential, but highly inefficient, even in vivo, with individual subunits entering the nucleolus rather than the preassembled holoenzyme (Dundr et al., 2002).

\section{ASSEMBLY FACTORS ARE REQUIRED FOR THE ASSEMBLY PROCESSES OF RNA POLYMERASES}

In yeast, the assembly of RNA pols and their transport to the nucleus require the action of assembly and/or transport factors, most of which are conserved in human cells. The pre-existing knowledge about their role in the assembly of yeast RNA pols and their comparison with that in humans are summarized in Table 1. How these factors act to mediate the sequential assembly of RNA pols and the nuclear transport is shown in Figure 1.

\section{R2TP/Prefoldin-like}

R2TP was initially identified in yeast as an Hsp90- associated multiprotein complex (R2TP-Hsp90 complex) (Zhao et al., 2005). This complex is well conserved from yeast to humans (Zhao et al., 2005; Boulon et al., 2008). Yeast R2TP components Rvb1/Rvb2 associate independently with whole RNA pol II and the Hsp90 complex (Lakshminarasimhan et al., 2016). R2TP subunits have also been detected in the polysomes interacting with newly synthesized Rpb1 subunits (Villanyi et al., 2014). Furthermore, the co-translational assembly between R2TP, Hsp90, and Rpb1 is mediated by Not5 (Villanyi et al., 2014). Similarly, in human cells, the co-chaperone R2TP complex works with HSP90 in the activation and assembly of several macromolecular complexes, including RNA pol II (Boulon et al., 2010). Proteomic analyses in human cells have evidenced the presence of RPB1-RPB8 dimer (RPB8 also called RPABC3) that interacts with the full R2TP/PFDL complex (Boulon et al., 2010). Human R2TP subunit RPAP3 delivers unassembled RPB1 to HSP90 and also associates with the largest subunits RPA190 (also called RPA1) (Boulon et al., 2010) and RPC160 (also called RPC1) of the free RNA pol I and RNA pol III (Jeronimo et al., 2007), respectively. These data suggest that R2TP subunit RPAP3 may be involved in the assembly of all three RNA pols (Boulon et al., 2010).

The human R2TP complex interacts with components of the prefoldin (PFD) complex (PFDN2 and PFDN6) and the prefoldin-like complex (URI, UXT, and PDRG1) to form the R2TP/prefoldin-like complex (R2TP/PFDL) (Cloutier et al., 2009; Cloutier and Coulombe, 2010; Martínez-Fernández et al., 2018). URI yeast ortholog Bud27 interacts with prefoldin subunits 2 and 6 (Pfd2 and Pfd6) and with the RNA pols common subunit Rpb5 and plays a role in the cytoplasmic assembly of RNA pol I, II, and III (Cloutier et al., 2009; Cloutier and Coulombe, 2010; Mirón-García et al., 2013; Martínez-Fernández et al., 2018).

These data suggest that R2TP, in both yeast and human, participates in the assembly of subassembly complex Rpb1 to the rest of the RNA pol II enzyme and likely occurs for RNA pol I and III.

\section{HSP90}

Yeast Hsp90 and human HSP90 are well-conserved molecular chaperones that participate in protein folding and avoid the non-specific aggregation of non-native proteins (Pearl and Prodromou, 2006; Wandinger et al., 2008; Taipale et al., 2010; Makhnevych and Houry, 2012; Schopf et al., 2017).

As indicated above, yeast Hsp90 associates with RNA pol II (Lakshminarasimhan et al., 2016) and human HSP90 and R2TP/PFDL mediate the assembly of RNA pol II through the interaction with the RPB1 subcomplex (Boulon et al., 2010, 2012; Makhnevych and Houry, 2012). Interestingly, yeast Rpa135 (RNA pol I) and Rpc40 (RNA pol III) feature among Hsp90 clients (McClellan et al., 2007). These data suggest that Hsp90 could mediate the assembly of RNA pol I, II, and III. HSP90 is required for RPB1 stabilization through most of the assembly pathway, particularly for the RPB1 subunit association with RPB8 (also called RPABC3) and RPB5 (also called RPABC1) and also with the RPB2-RPB3-RPB10-RPB11-RPB12 subcomplex (also called RPB2-RPB3-RPABC5-RPB11-a and RPABC4), by facilitating the assembly of the complete enzyme (Boulon et al., 2010, 2012). Therefore, HSP90/R2TP could mediate a quality control mechanism for RNA pol II formation by ensuring its correct assembly before its nuclear import (Boulon et al., 2012). Interestingly, and as previously demonstrated for yeast (Lakshminarasimhan et al., 2016), proteomic analyses have also revealed not only RPB1 but also RPA190 (also called RPA1) and RPC160 (also called RPC1) to be HSP90/R2TP interactors, which also suggests their role in the assembly of RNA pol I and III (Boulon et al., 2010).

\section{Bud27}

Bud27, and its human ortholog URI, are members of the PFD family of the ATP-independent molecular chaperones considered to function as scaffold proteins capable of assembling additional members of the PFD family in both human and yeast (Gstaiger et al., 2003; Martínez-Fernández and Navarro, 2018). Bud27 contacts the Pfd6 and Pfd2 components of the PFD/GimC complex (Gstaiger et al., 2003; Mirón-García et al., 2013), whereas URI contacts the PFD complex (Gstaiger et al., 2003).

Both Bud27 and URI interact with Rpb5, a common subunit of eukaryotic RNA pols (Dorjsuren et al., 1998; Gstaiger et al., 2003; Mirón-García et al., 2013; Martínez-Fernández and Navarro, 2018). Bud27 mediates the cytoplasmic assembly of the three RNA pols in Saccharomyces cerevisiae in an Rpb5-dependent manner before nuclear translocation, which probably occurs similarly for human URI, at least for RNA pol II (Mirón-García et al., 2013). Furthermore, proteomic analyses reveal URI to be an R2TP/PFDL component involved in, at least, RNA pol II assembly in human (Cloutier et al., 2009; Cloutier and Coulombe, 2010; Martínez-Fernández et al., 2018).

Bud27 shuttles between the nucleus and the cytoplasm (Mirón-García et al., 2013) and participates in the transcription mediated by the three RNA pols (Mirón-García et al., 2014; Vernekar and Bhargava, 2015; Martínez-Fernández et al., 2020). These results suggest that Bud27 could be imported to the nucleus in association with RNA pols and then remain associated with 
TABLE 1 | Assembly factors.

\begin{tabular}{llll}
\hline \multicolumn{2}{c}{ Assembly factor } & Eukaryotic RNA pol & References \\
\cline { 1 - 2 } S. cerevisiae & Human & & \\
\cline { 1 - 2 } R2TP complex & R2TP/prefoldin-like complex & RNA pol II. Also suggested for RNA pol I and III & Boulon et al., 2010 \\
Hsp90 & HSP90 & RNA pol II. Also suggested for RNA pol I and III & Boulon et al., 2010 \\
Npa3 & GPN1/RPAP4/XAB1/MBDin & RNA pol II & Forget et al., 2010; Staresincic et al., 2011; Niesser et al., 2015 \\
Gpn2 & GPN2 & RNA pol II and III & Staresincic et al., 2011; Minaker et al., 2013; Zeng et al., 2018 \\
Gpn3 & GPN3/Parcs & RNA pol II and III & Calera et al., 2011; Minaker et al., 2013; Liu et al., 2020 \\
Rba50 & RPAP1 & RNA pol II & Jeronimo et al., 2004; Zeng et al., 2018; Liu et al., 2020 \\
Rtr1 & RPAP2 & RNA pol II & Forget et al., 2013; Gómez-Navarro and Estruch, 2015 \\
Bud27 & URI & RNA pol I, II, III & Mirón-García et al., 2013; Vernekar and Bhargava, 2015 \\
Rtp1 & & RNA pol II & Gómez-Navarro et al., 2013 \\
Rbs1 & & RNA pol III & Cieśla et al., 2015 \\
Iwr1 & RNA pol II & Czeko et al., 2011
\end{tabular}

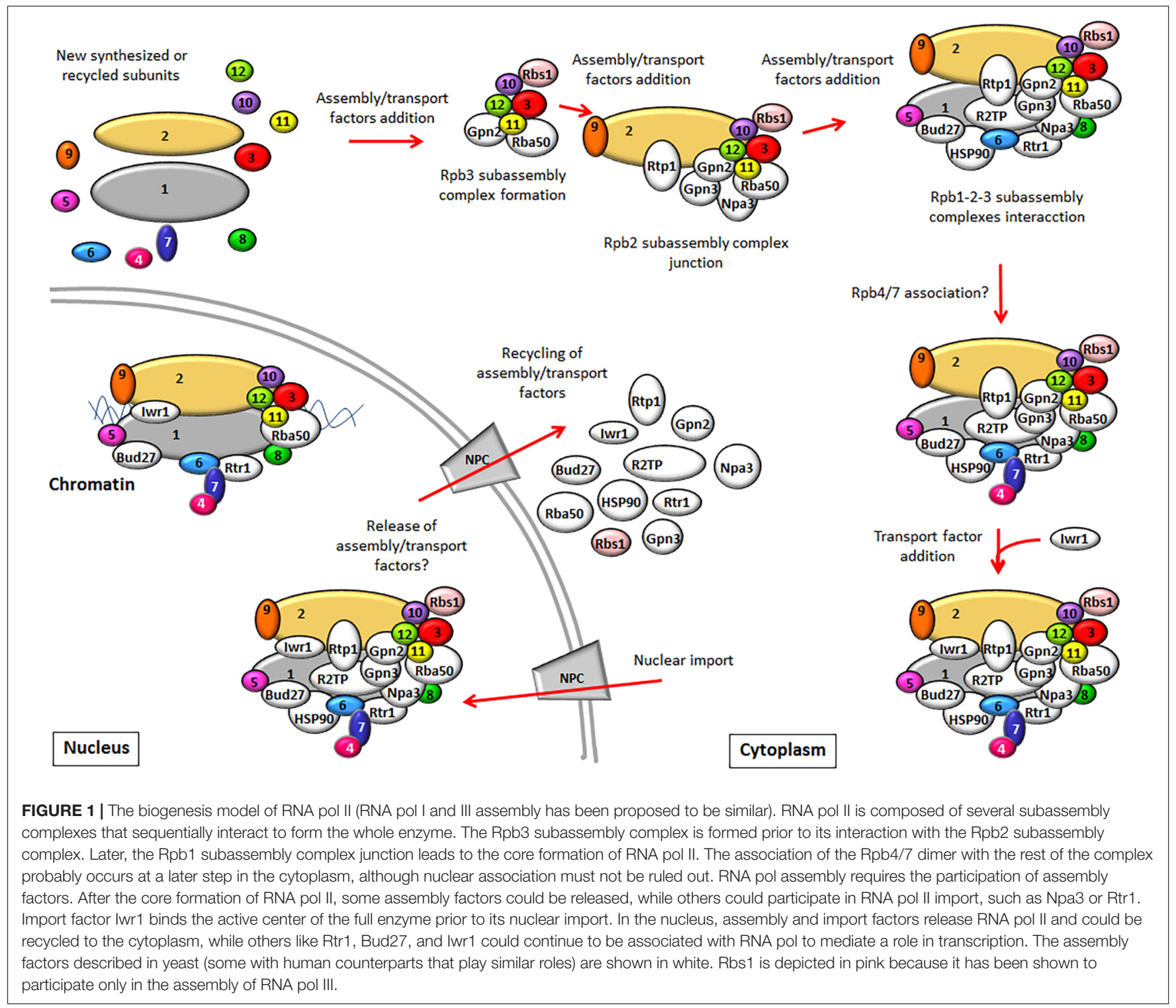


the transcriptional complexes, probably by a tripartite interaction with Rpb5 and remodeler complexes like RSC (Mirón-García et al., 2014; Vernekar and Bhargava, 2015).

\section{The GPN-Loop GTPase Family}

GPN-loop GTPase proteins, highly conserved from archaea to humans, contain a highly conserved GPN-loop motif of GlyPro-Asn inserted into the GTPase core-fold that functions in GTP hydrolysis (Gras et al., 2007). In yeast, three GPN-loop GTPase, Npa3, Gpn2, and Gpn3 (GPN1, GPN2, and GPN3 in human, respectively), have been described, which evolved from a single archaeal counterpart (Gras et al., 2007; Forget et al., 2010; Carre and Shiekhattar, 2011; Staresincic et al., 2011; Minaker et al., 2013).

The three yeast and human small GTPases have been described to participate in RNA pol II assembly and/or transport to the nucleus (Forget et al., 2010; Carre and Shiekhattar, 2011; Staresincic et al., 2011; Minaker et al., 2013; Niesser et al., 2015; Li et al., 2018; Zeng et al., 2018; Liu et al., 2020). Furthermore, interactions among the three yeast and human members of the GPN-loop GTPase family have been detected (Uetz et al., 2000; Boulon et al., 2010; Forget et al., 2010; Carre and Shiekhattar, 2011; Staresincic et al., 2011; Minaker et al., 2013; Liu et al., 2020). A more detailed overview of these proteins is shown below.

\section{Npa3}

Npa3 interacts with RNA pol II and the R2TP complex in yeast (Forget et al., 2010; Niesser et al., 2015). Similarly, the human Npa3 ortholog, GPN1, has been described as an RNA pol II-associated protein that interacts with not only the complex R2TP/PFDL, and the cytosolic chaperonin, CCT, but also with other proteins involved in protein assembly and/or folding (Jeronimo et al., 2007; Forget et al., 2010). Npa3 depletion leads to the cytoplasmic accumulation of Rpb1 and Rpb3 (Staresincic et al., 2011). Cytoplasmic Rpb1 accumulation is also observed in mutants of the Npa3 GTP-binding domain or GPN motifs (similarly for human GPN1) (Forget et al., 2010; Carre and Shiekhattar, 2011; Staresincic et al., 2011). Nevertheless, Rpb3 immunoprecipitation evidences that $\mathrm{Npa} 3$ coordinates not only with Gpn3 but also with Rba50 (described in detail in the next paragraph), for the correct association of Rpb1 and Rpb2 with the Rpb3 subcomplex, likely in the cytoplasm (Liu et al., 2020). These data point to a major role of this protein in the cytoplasmic assembly of RNA pol II as has been previously proposed (Niesser et al., 2015). In line with this, two-hybrid assays have shown that the Rpb2 subunit contacts Npa3 and Rba50, which suggests that both proteins may coordinate the Rpb2 subcomplex-dependent assembly of RNA pol II (Liu et al., 2020). In fact, Npa3 is found mainly in the cytoplasm (Huh et al., 2003), but it contains a nuclear export sequence (NES) (Staresincic et al., 2011) that is also conserved in its human ortholog GPN1 (Reyes-Pardo et al., 2012). Npa3 and, similarly human GPN1, translocates to the cytoplasm by the action of the Xpo1/Crm1 pathway (Forget et al., 2010; Carre and Shiekhattar, 2011; Staresincic et al., 2011) and has been proposed to participate mainly in the nuclear import of RNA pol II (Forget et al., 2010; Carre and Shiekhattar, 2011; Staresincic et al., 2011). In line with data in yeast, two-hybrid experiments in human cells have demonstrated the interaction between GPN1 and Rba50 human ortholog RPAP1 and also between GPN1 and RPB2 (Liu et al., 2020). These findings suggest that the role proposed for Npa3 in the assembly of RNA pol II is also conserved in human cells.

\section{Gpn2}

Gpn2 is another GPN-loop GTPase family member (Alonso et al., 2013). Similar to Gpn1 and Gpn3, Gpn2 loss-of-function leads to altered cytoplasmic RNA pol II localization, which suggests a role for Gpn2 in the transport of RNA pol II to the nucleus (Staresincic et al., 2011; Minaker et al., 2013; Zeng et al., 2018; Liu et al., 2020). Gpn2 physically interacts with Rba50 and Rpb12 by cooperating to assemble the Rpb3 subcomplex prior to its association with Rpb1 and Rpb2 (Zeng et al., 2018). Yeast gpn2 mutants show genetic interactions with RNA pol I and III mutants (Minaker et al., 2013). Although gpn2 mutants affect the localization of both RNA pol II and III, they do not mislocalize RNA pol I subunits (Minaker et al., 2013). These results suggest that Gpn2 acts not only in RNA pol II assembly but also in the RNA pol I and III assembly process.

\section{Gpn3}

Gpn3 has been proposed to mediate RNA pols biogenesis, assembly, and transport to the nucleus (Minaker et al., 2013; Liu et al., 2020) and has been demonstrated to form a stable complex with Gpn1 in both yeast and human (Carre and Shiekhattar, 2011; Cristóbal-Mondragón et al., 2019; Liu et al., 2020). In addition, yeast gpn 3 mutants mislocalize RNA pol II and III subunits, which suggests a role for Gpn3 (and Gpn2) in not only RNA pol II but also in RNA pol III assembly and/or transport (Minaker et al., 2013). Gpn3 has been proposed to act upstream of import factor Iwr1 during RNA pol II biogenesis (Minaker et al., 2013). Proteomic analyses have evidenced the human GPN3, as well as GPN1 and GPN2, to be an interactor of the RNA pol II subunits (Forget et al., 2010; Calera et al., 2011). The human GPN1/GPN3 complex associates with RNA pol II in both the nucleus and the cytoplasm (Carre and Shiekhattar, 2011) and specifically binds RPB7 (likely the RPB7/RPB4 dimer) and the C-terminal domain (CTD) of RPB1 in vitro (Carre and Shiekhattar, 2011). Furthermore, the depletion of human GPN3 or GPN1 by small interfering RNAs (siRNAs) leads to RPB1 cytoplasmic accumulation (Calera et al., 2011; Carre and Shiekhattar, 2011). In line with a role for GPN3 in RNA pol II transport to the nucleus, the Q279* mutation of GPN3, related to cancer, has been described to lead to GPN3 entering the cell nucleus and inhibiting GPN1 nuclear export (Barbosa-Camacho et al., 2017).

By taking these data collectively, the GPN-loop GTPase family would act by favoring the assembly and/or transport of the three RNA pols in yeast, and these roles could be conserved in human cells.

\section{Rba50/RPAP1}

Rba50 has been described as a cytoplasmic protein that interacts not only with the Rpb10 subunit in two-hybrid screening (Ito et al., 2001; Huh et al., 2003) but also with Rpb2, Rpb3, and Rpb11 in TAP-tagging analyses (Hazbun et al., 2003). 
In yeast, physical and functional interactions have been demonstrated between Rba50 and the small GTPases Gpn2 and Npa3, and these interactions are conserved in human and Arabidopsis (Muñoz et al., 2017; Li et al., 2018; Zeng et al., 2018; Liu et al., 2020).

Recent studies propose the coordinated action between Rba50 and Gpn2 in the Rpb3 subcomplex assembly prior to its association with the Rpb2 subassembly complex (Zeng et al., 2018). The association among Rba50, Rpb3, Rpb10, and Rpb11 and between Gpn2 and Rpb12 would allow the Rpb3 subassembly complex formation (Zeng et al., 2018). Although yeast rba503 mutant cells affect Rpb1 distribution, no interactions between Rpb1 and Rba50 or Gpn2 have been identified, which suggests that Rba50 and Gpn2 only transiently associate with the Rpb3 subcomplex and dissociate once Rpb1 is associated during the assembly of RNA pol II (Zeng et al., 2018). The recent observation that Rba50 and Npa3 not only interact but also target Rpb2 during the biogenesis of RNA pol II suggests that Rba50 also associates with Npa3 by increasing its affinity to Rpb2 to facilitate Rpb2 assembly to the previously formed Rpb3 subassembly complex (Liu et al., 2020). As previously indicated, human Rba50 ortholog RPAP1 interacts with GPN1, which associates with RPB2 (Liu et al., 2020). RPAP1 enters the nucleus and has been proposed to be required for the transcription of cell identity genes (those genes regulating the developmental process and fibroblastic/mesenchymal identity) by operating at the interface between the Mediator and RNA pol II (Lynch et al., 2018).

Based on yeast and human data, some authors propose that Rba50 (and probably its human ortholog RPAP1) functions by favoring a platform for other assembly factors like Gpn2 and $\mathrm{Npa} 3$ to sequentially mediate the association of RNA pol II subassembly complexes (Liu et al., 2020).

\section{Rtr1/RPAP2}

Rtr1 ("regulator of transcription" 1) has been described as a phosphorylated RNA pol II interactor by acting as an S5-P CTD phosphatase during the transition from the initiation of the transcription to elongation in vivo (Gibney et al., 2008; Mosley et al., 2009, 2013; Hsu et al., 2014; Smith-Kinnaman et al., 2014; Hunter et al., 2016). Additional roles have been proposed for Rtr1 in transcription and mRNA stability (Mosley et al., 2013; Hsu et al., 2014; Hodko et al., 2016; Victorino et al., 2020) (our unpublished data).

It has been proposed that Rtr1 acts as a nuclear RNA pol II import factor as RTR1 deletion causes cytoplasmic accumulation of Rpb1 and Rpb2 (Gómez-Navarro and Estruch, 2015). Rtr1 shuttles between the nucleus and cytoplasm in a Crm1-dependent manner (Gibney et al., 2008) and interacts with the nucleocytoplasmic transport protein Ran (a small Ras-like GTPase) (Braunwarth et al., 2003). The deletion of RTR1 paralog RTR2 does not cause mislocalization of Rpb1, but it increases in the double rtr1 rtr2 mutant. These findings suggest that both proteins may play a redundant role in RNA pol II import (Gómez-Navarro and Estruch, 2015). Interestingly, Rtr1 also copurifies with the GTPases Gpn3 and Npa3 (Mosley et al., 2013; Smith-Kinnaman et al., 2014), which are proteins that have also been implicated in the nuclear import of RNA pol II (Staresincic et al., 2011).

Human Rtr1 ortholog RPAP2 has been proposed to act as an RNA pol II import factor, given its silencing results in RPB1 cytoplasmic accumulation (Forget et al., 2013). RPAP2 interacts with RNA pol II through its nuclear retention domain in vitro (Forget et al., 2013) and directly binds the RPB6 subunit of the enzyme (Wani et al., 2014). In addition, proteomic analyses of human subassembly complexes have identified that RPAP2 is preferentially associated with the free RPB3 and RPB1 subunits (Boulon et al., 2010), which suggests a role for RPAP2 in the biogenesis of RNA pol II. Furthermore, RPAP2 shuttles to the cytoplasm in association with GPN1 (Forget et al., 2013; Guerrero-Serrano et al., 2017). As in yeast, this evidences the relationship between RPAP2 and small GTPases. Interestingly, Arabidopsis Rtr1 ortholog RIMA interacts with MINIYO (the Rba50 yeast ortholog) (Muñoz et al., 2017).

By considering the role of Rtr1 in only the transcription of RNA pol II (Gibney et al., 2008; Mosley et al., 2009; Hsu et al., 2014; Victorino et al., 2020) and the above data, we speculate that this protein is specific to the assembly and/or transport of RNA pol II in yeast and also in other organisms.

\section{Rtp1}

Rtp1 has been proposed to be an important factor for nuclear RNA pol II localization via Iwr1-independent pathways (GómezNavarro et al., 2013). In fact, it has been suggested to participate in transporting RNA pol II through the nuclear pore complex (Gómez-Navarro et al., 2013; Gómez-Navarro and Estruch, 2015). Rtp1 physically interacts with R2TP complex components and also with several RNA pol II subunits (Gómez-Navarro et al., 2013). Mass spectrometry data suggest that Rtp1 can facilitate the interaction between subassembly complexes Rpb2 and Rpb3, and their later interaction with the subassembly complex Rpb1 for the assembly of RNA pol II (Gómez-Navarro et al., 2013). RTP1 gene depletion in yeast leads to the cytoplasmic accumulation of Rpb1 and Rpb2 (Gómez-Navarro et al., 2013). Interestingly, the fact that the $r t p 1$ mutant shows no clear cytoplasmic accumulation of small RNA pol II subunits, such as Rpb3 and Rpb11, suggests the existence of passive nuclear diffusion of small RNA pol II subunits (Gómez-Navarro and Estruch, 2015).

\section{Iwr1}

Iwr1 was initially reported as a protein that interacts with almost every RNA pol II subunit to regulate the transcription of some genes (Gavin et al., 2002; Peiro-Chova and Estruch, 2009) and was later reported as being important for preinitiation complex formation by all three nuclear RNA pols in S. cerevisiae (Esberg et al., 2011). It has been proposed that the main pathway for the nuclear RNA pol II import involves yeast Iwr1 (Czeko et al., 2011). Iwr1 binds the cleft of the active center of RNA pol II once the enzyme is fully assembled and uses its NLS signal to direct the nuclear import of RNA pol II (Czeko et al., 2011). Iwr1 is displaced from active RNA pol II in the nucleus, which facilitates its export and recycling (Czeko et al., 2011; Wild and Cramer, 2012). Nevertheless, iwr $1 \Delta$ mutant strains show cytoplasmic 
accumulation of both Rpb1 and Rpb3 (Czeko et al., 2011), which suggests interferences with the assembly of RNA pol II.

In addition to Iwr1-dependent nuclear RNA pol II import, Iwr1-independent import pathways have been proposed as mechanisms that maintain cell viability when the main pathways are blocked, which involves the nuclear import of individual or partially assembled subunits, even by diffusion (Gómez-Navarro and Estruch, 2015).

\section{Rbs1}

Rbs1 was originally identified as a PAS kinase suppressor in a genetic high-copy suppressor study (Rutter et al., 2002). More recently, a role for Rbs1 in RNA pol III assembly and transport to the nucleus has been proposed (Cieśla et al., 2015). This role for Rbs1 has also been described in another article published in the same issue by Boguta and Turowski (in press). Rbs1 physically interacts not only with several RNA pol III subunits, such as Rpc19 and Rpc40 but also with the RNA pol common subunit Rpb5 (Cieśla et al., 2015). Rbs1 has been described as a Crm1 interactor, and it shuttles between the nucleus and the cytoplasm. Accordingly, it has been suggested to likely interact with the RNA pol III complex to mediate its nuclear translocation (Cieśla et al., 2015). Rbs1 has also been proposed to mediate the biogenesis of RNA pol III by controlling the steady-state levels of RPB10 mRNA by interacting with its $3^{\prime}$ UTR region (Cieśla et al., 2020).

\section{CONCLUDING REMARKS}

Despite the pre-existing knowledge about RNA pols assembly in yeast and their transport to the nucleus, mainly focused

\section{REFERENCES}

Alonso, B., Beraud, C., Meguellati, S., Chen, S. W., Pellequer, J. L., Armengaud, J., et al. (2013). Eukaryotic GPN-loop GTPases paralogs use a dimeric assembly reminiscent of archeal GPN. Cell Cycle 12, 463-472. doi: 10.4161/cc.23367

Armache, K. J., Kettenberger, H., and Cramer, P. (2003). Architecture of initiationcompetent 12-subunit RNA polymerase II. Proc. Natl. Acad. Sci. U.S.A. 100, 6964-6968. doi: 10.1073/pnas.1030608100

Armache, K. J., Mitterweger, S., Meinhart, A., and Cramer, P. (2005). Structures of complete RNA polymerase II and its subcomplex, Rpb4/7. J. Biol. Chem. 280, 7131-7134. doi: 10.1074/jbc.m413038200

Barbosa-Camacho, A. A., Méndez-Hernández, L. E., Lara-Chacón, B., PeñaGómez, S. G., Romero, V., González-González, R., et al. (2017). The Gpn3 Q279* cancer-associated mutant inhibits Gpn1 nuclear export and is deficient in RNA polymerase II nuclear targeting. FEBS Lett. 591, 3555-3566. doi: 10.1002/1873-3468.12856

Boulon, S., Bertrand, E., and Pradet-Balade, B. (2012). HSP90 and the R2TP co-chaperone complex: building multi-protein machineries essential for cell growth and gene expression. RNA Biol. 9, 148-154. doi: 10.4161/rna. 18494

Boulon, S., Marmier-Gourrier, N., Pradet-Balade, B., Wurth, L., Verheggen, C., Jady, B. E., et al. (2008). The Hsp90 chaperone controls the biogenesis of L7Ae RNPs through conserved machinery. J. Cell Biol. 180, 579-595. doi: 10.1083/jcb. 200708110

Boulon, S., Pradet-Balade, B., Verheggen, C., Molle, D., Boireau, S., Georgieva, M., et al. (2010). HSP90 and its R2TP/Prefoldin-like cochaperone are involved in the cytoplasmic assembly of RNA polymerase II. Mol. Cell 39, 912-924. doi: 10.1016/j.molcel.2010.08.023 on RNA pol II and likely well-conserved for human RNA pol II, many interesting questions still need answers: although the assembly of RNA pols in eukaryotes seems to be similar to that in bacteria, why do RNA pols-specific processes exist? Why do RNA pol-specific assembly factors exist, while others seem to be general? Can different and additional mechanisms act for the assembly of RNA pols in yeast and other eukaryotes? Which mechanisms account for the nuclear transport of different RNA pols?

Resolving these important questions and others must be the goal of future studies, to help us better understand the mechanisms governing the assembly and nuclear transport of different RNA pols.

\section{AUTHOR CONTRIBUTIONS}

All authors wrote and reviewed this manuscript.

\section{FUNDING}

This work was supported by grants from the Spanish Ministry of Economy and Competitiveness (MINECO) and ERDF (BFU2016-77728-C3-2-P), the Spanish Ministry of Science and Innovation (MICINN) and ERDF (RED2018-102467-T), the Junta de Andalucía-Universidad de Jaén (FEDER-UJA 1260360), and the Junta de Andalucía (BIO258) to FN. AG-G was financed by the Universidad de Jaen, MINECO, and ERDF funds (BFU2016-77728-C3-2-P to FN). FG-S is a recipient of a predoctoral fellowship from the Universidad de Jaen.

Braunwarth, A., Fromont-Racine, M., Legrain, P., Bischoff, F. R., Gerstberger, T., Hurt, E., et al. (2003). Identification and characterization of a novel RanGTPbinding protein in the yeast Saccharomyces cerevisiae. J. Biol. Chem. 278, 15397-15405. doi: 10.1074/jbc.m210630200

Briand, J. F., Navarro, F., Rematier, P., Boschiero, C., Labarre, S., Werner, M., et al. (2001). Partners of Rpb8p, a small subunit shared by yeast RNA polymerases I. II and III. Mol. Cell Biol. 21, 6056-6065. doi: 10.1128/mcb.21.17.6056-6065. 2001

Calera, M. R., Zamora-Ramos, C., Araiza-Villanueva, M. G., Moreno-Aguilar, C. A., Peña-Gómez, S. G., Castellanos-Terán, F., et al. (2011). Parcs/Gpn3 is required for the nuclear accumulation of RNA polymerase II. Biochim. Biophys. Acta Mol. Cell Res. 1813, 1708-1716. doi: 10.1016/j.bbamcr.2011.07.005

Carre, C., and Shiekhattar, R. (2011). Human GTPases associate with RNA polymerase II To mediate its nuclear import. Mol. Cell Biol. 31, 3953-3962. doi: $10.1128 / \mathrm{mcb} .05442-11$

Cieśla, M., Makała, E., Płonka, M., Bazan, R., Gewartowski, K., Dziembowski, A., et al. (2015). Rbs1, a new protein implicated in RNA polymerase III biogenesis in yeast Saccharomyces cerevisiae. Mol. Cell. Biol. 35, 1169-1181. doi: 10.1128/ MCB.01230- 14

Cieśla, M., Turowski, T. W., Nowotny, M., Tollervey, D., and Boguta, M. (2020). The expression of Rpb10, a small subunit common to RNA polymerases, is modulated by the R3H domain-containing Rbs1 protein and the Upf1 helicase. Nucleic Acids Res. 48, 12252-12268. doi: 10.1093/nar/gkaa1069

Cloutier, P., Al-Khoury, R., Lavallee-Adam, M., Faubert, D., Jiang, H., Poitras, C., et al. (2009). High-resolution mapping of the protein interaction network for the human transcription machinery and affinity purification of RNA polymerase II-associated complexes. Methods 48, 381-386. doi: 10.1016/j. ymeth.2009.05.005 
Cloutier, P., and Coulombe, B. (2010). New insights into the biogenesis of nuclear RNA polymerases? Biochem. Cell Biol. 88, 211-221.

Corden, J. (2011). Going nuclear: transcribers in transit. Mol. Cell 42, 143-145. doi: 10.1016/j.molcel.2011.04.001

Cramer, P. (2002). Multisubunit RNA polymerases. Curr. Opin. Struct. Biol. 12, 89-97. doi: 10.1016/s0959-440x(02)00294-4

Cramer, P. (2019). Eukaryotic transcription turns 50. Cell 179, 808-812. doi: 10. 1016/j.cell.2019.09.018

Cristóbal-Mondragón, G. R., Lara-Chacón, B., Santiago, Á, De-la-Rosa, V., González-González, R., Muñiz-Luna, J. A., et al. (2019). FRET-based analysis and molecular modeling of the human GPN-loop GTP ases 1 and 3 heterodimer unveils a dominant-negative protein complex. FEBS J. 286, 47974818. doi: $10.1111 /$ febs. 14996

Czeko, E., Seizl, M., Augsberger, C., Mielke, T., and Cramer, P. (2011). Iwr1 directs RNA polymerase II nuclear import. Mol. Cell 42, 261-266. doi: 10.1016/j. molcel.2011.02.033

Dieci, G., Conti, A., Pagano, A., and Carnevali, D. (2012). Identification of RNA polymerase III-transcribed genes in eukaryotic genomes. Biochim. Biophys. Acta 1829, 296-305. doi: 10.1016/j.bbagrm.2012.09.010

Dorjsuren, D., Lin, Y., Wei, W., Yamashita, T., Nomura, T., Hayashi, N., et al. (1998). RMP, a novel RNA polymerase II subunit 5-interacting protein, counteracts transactivation by hepatitis B virus X protein. Mol. Cell Biol. 18, 7546-7555. doi: $10.1128 / \mathrm{mcb} .18 .12 .7546$

Dundr, M., Hoffmann-Rohrer, U., Hu, Q., Grummt, I., Rothblum, L. I., Phair, R. D., et al. (2002). A kinetic framework for a mammalian RNA polymerase in vivo. Science 298, 1623-1626. doi: 10.1126/science.1076164

Esberg, A., Moqtaderi, Z., Fan, X., Lu, J., Struhl, K., and Bystrom, A. (2011). Iwr1 protein is important for preinitiation complex formation by all three nuclear RNA polymerases in Saccharomyces cerevisiae. PLoS One 6:e20829. doi: 10.1371/journal.pone.0020829

Fernández-Tornero, C., Bottcher, B., Rashid, U. J., and Muller, C. W. (2011). Analyzing RNA polymerase III by electron cryomicroscopy. RNA Biol. 8, 760-765. doi: 10.4161/rna.8.5.16021

Fernández-Tornero, C., Moreno-Morcillo, M., Rashid, U. J., Taylor, N. M., Ruiz, F. M., Gruene, T., et al. (2013). Crystal structure of the 14-subunit RNA polymerase I. Nature 502, 644-649.

Forget, D., Lacombe, A. A., Cloutier, P., Al-Khoury, R., Bouchard, A., LavalleeAdam, M., et al. (2010). The protein interaction network of the human transcription machinery reveals a role for the conserved GTPase RPAP4/GPN1 and microtubule assembly in nuclear import and biogenesis of RNA polymerase II. Mol. Cell Proteomics 9, 2827-2839. doi: 10.1074/mcp.m110.003616

Forget, D., Lacombe, A. A., Cloutier, P., Lavallee-Adam, M., Blanchette, M., and Coulombe, B. (2013). Nuclear import of RNA polymerase II is coupled with nucleocytoplasmic shuttling of the RNA polymerase II-associated protein 2. Nucleic Acids Res. 41, 6881-6891. doi: 10.1093/nar/gkt455

Garrido-Godino, A. I., Garcíaa-López, M. C., and Navarro, F. (2013). Correct assembly of RNA polymerase II depends on the foot domain and is required for multiple steps of transcription in Saccharomyces cerevisiae. Mol. Cell Biol. 33, 3611-3626. doi: 10.1128/mcb.00262-13

Gavin, A. C., Bosche, M., Krause, R., Grandi, P., Marzioch, M., Bauer, A., et al. (2002). Functional organization of the yeast proteome by systematic analysis of protein complexes. Nature 415, 141-147.

Gibney, P. A., Fries, T., Bailer, S. M., and Morano, K. A. (2008). Rtr1 is the Saccharomyces cerevisiae homolog of a novel family of RNA polymerase IIbinding proteins. Eukaryot. Cell 7, 938-948. doi: 10.1128/ec.00042-08

Gómez-Navarro, N., and Estruch, F. (2015). Different pathways for the nuclear import of yeast RNA polymerase II. Biochim. Biophys. Acta Gene Regul. Mech. 1849, 1354-1362. doi: 10.1016/j.bbagrm.2015.10.003

Gómez-Navarro, N., Peiro-Chova, L., Rodríguez-Navarro, S., Polaina, J., and Estruch, F. (2013). Rtp1p is a karyopherin-like protein required for RNA polymerase II biogenesis. Mol. Cell Biol. 33, 1756-1767. doi: 10.1128/MCB. 01449-12

Gras, S., Chaumont, V., Fernandez, B., Carpentier, P., Charrier-Savournin, F., Schmitt, S., et al. (2007). Structural insights into a new homodimeric self-activated GTPase family. EMBO Rep. 8, 569-575. doi: 10.1038/sj.embor. 7400958

Gstaiger, M., Luke, B., Hess, D., Oakeley, E. J., Wirbelauer, C., Blondel, M., et al. (2003). Control of nutrient-sensitive transcription programs by the unconventional prefoldin URI. Science 302, 1208-1212. doi: 10.1126/science. 1088401

Guerrero-Serrano, G., Castanedo, L., Cristóbal-Mondragón, G. R., MontalvoArredondo, J., Riego-Ruiz, L., DeLuna, A., et al. (2017). Npa3/ScGpn1 carboxyterminal tail is dispensable for cell viability and RNA polymerase II nuclear targeting but critical for microtubule stability and function. Biochim. Biophys. Acta Mol. Cell Res. 1864, 451-462. doi: 10.1016/j.bbamcr.2016.12.010

Haag, J. R., Brower-Toland, B., Krieger, E. K., Sidorenko, L., Nicora, C. D., Norbeck, A. D., et al. (2014). Functional diversification of maize RNA polymerase IV and V subtypes via alternative catalytic subunits. Cell Rep. 9, 378-390. doi: 10.1016/j.celrep.2014.08.067

Haag, J. R., and Pikaard, C. S. (2011). Multisubunit RNA polymerases IV and V: purveyors of non-coding RNA for plant gene silencing. Nat. Rev. Mol. Cell Biol. 12, 483-492. doi: $10.1038 / \mathrm{nrm} 3152$

Hardeland, U., and Hurt, E. (2006). Coordinated nuclear import of RNA polymerase III subunits. Traffic 7, 465-473. doi: 10.1111/j.1600-0854.2006. 00399.x

Hazbun, T. R., Malmstrom, L., Anderson, S., Graczyk, B. J., Fox, B., Riffle, M., et al. (2003). Assigning function to yeast proteins by integration of technologies. Mol. Cell 12, 1353-1365. doi: 10.1016/s1097-2765(03)00476-3

Hodko, D., Ward, T., and Chanfreau, G. (2016). The Rtr1p CTD phosphatase autoregulates its mRNA through a degradation pathway involving the REX exonucleases. RNA 22, 559-570. doi: 10.1261/rna.055723.115

Hsu, P. L., Yang, F., Smith-Kinnaman, W., Yang, W., Song, J.-E., Mosley, A. L., et al. (2014). Rtr1 is a dual specificity phosphatase that dephosphorylates Tyr1 and Ser5 on the RNA polymerase II CTD. J. Mol. Biol. 426, 2970-2981. doi: 10.1016/j.jmb.2014.06.010

Huh, W. K., Falvo, J. V., Gerke, L. C., Carroll, A. S., Howson, R. W., Weissman, J. S., et al. (2003). Global analysis of protein localization in budding yeast. Nature $425,686-691$.

Hunter, G. O., Fox, M. J., Smith-Kinnaman, W. R., Gogol, M., Fleharty, B., and Mosley, A. L. (2016). Phosphatase Rtr1 regulates global levels of serine 5 RNA polymerase II C-terminal domain phosphorylation and cotranscriptional histone methylation. Mol. Cell. Biol. 36, 2236-2245. doi: 10.1128/mcb.00 870-15

Ishihama, A. (1981). Subunit of assembly of Escherichia coli RNA polymerase. Adv. Biophys. 14, 1-35. doi: 10.1016/0022-2836(91)90865-4

Ito, T., Chiba, T., Ozawa, R., Yoshida, M., Hattori, M., and Sakaki, Y. (2001). A comprehensive two-hybrid analysis to explore the yeast protein interactome. Proc. Natl. Acad. Sci. U.S.A. 98, 4569-4574. doi: 10.1073/pnas.061034498

Jeronimo, C., Forget, D., Bouchard, A., Li, Q., Chua, G., Poitras, C., et al. (2007). Systematic analysis of the protein interaction network for the human transcription machinery reveals the identity of the 7SK capping enzyme. Mol. Cell 27, 262-274. doi: 10.1016/j.molcel.2007.06.027

Jeronimo, C., Langelier, M. F., Zeghouf, M., Cojocaru, M., Bergeron, D., Baali, D., et al. (2004). RPAP1, a novel human RNA polymerase II-associated protein affinity purified with recombinant wild-type and mutated polymerase subunits. Mol. Cell Biol. 24, 7043-7058. doi: 10.1128/MCB.24.16.7043-7058.2004

Khatter, H., Vorlaender, M. K., and Mueller, C. W. (2017). RNA polymerase I and III: similar yet unique. Curr. Opin. Struct. Biol. 47, 88-94. doi: 10.1016/j.sbi. 2017.05.008

Kwapisz, M., Beckouet, F., and Thuriaux, P. (2008). Early evolution of eukaryotic DNA-dependent RNA polymerases. Trends Genet. 24, 211-215. doi: 10.1016/j. tig. 2008.02.002

Lakshminarasimhan, M., Boanca, G., Banks, C. A., Hattem, G. L., Gabriel, A. E., Groppe, B. D., et al. (2016). Proteomic and genomic analyses of the Rvb1 and Rvb2 interaction network upon deletion of R2TP complex components. Mol. Cell. Proteomics 15, 960-974. doi: 10.1074/mcp.m115.053165

Lane, L. A., Fernandez-Tornero, C., Zhou, M., Morgner, N., Ptchelkine, D., Steuerwald, U., et al. (2011). Mass spectrometry reveals stable modules in holo and apo RNA polymerases I and III. Structure 19, 90-100. doi: 10.1016/j.str. 2010.11.009

Li, Y., Yuan, Y., Fang, X., Lu, X., Lian, B., Zhao, G., et al. (2018). A role for MINIYO and QUATRE-QUART2 in the assembly of RNA polymerases II, IV, and V in Arabidopsis. Plant Cell 30, 466-480. doi: 10.1105/tpc.17.00380

Liu, X., Xie, D., Hua, Y., Zeng, P., Ma, L., and Zeng, F. (2020). Npa3 interacts with Gpn3 and assembly factor Rba50 for RNA polymerase II biogenesis. FASEB J. 34, 15547-15558. doi: 10.1096/fj.202001523r 
Lynch, C. J., Bernad, R., Calvo, I., Nóbrega-Pereira, S., Ruiz, S., Ibarz, N., et al. (2018). The RNA polymerase II factor RPAP1 is critical for mediator-driven transcription and cell identity. Cell Rep. 22, 396-410. doi: 10.1016/j.celrep.2017. 12.062

Makhnevych, T., and Houry, W. A. (2012). The role of Hsp90 in protein complex assembly. Biochim. Biophys. Acta Mol. Cell Res. 1823, 674-682. doi: 10.1016/j. bbamcr.2011.09.001

Martínez-Fernández, V., Cuevas-Bermúdez, A., Gutiérrez-Santiago, F., GarridoGodino, A. I., Rodríguez-Galán, O., Jordán-Pla, A., et al. (2020). Prefoldin-like Bud27 influences the transcription of ribosomal components and ribosome biogenesis in Saccharomyces cerevisiae. RNA 27, 1360-1379. doi: 10.1261/rna. 075507.120

Martínez-Fernández, V., Garrido-Godino, A. I., Cuevas-Bermúdez, A., and Navarro, F. (2018). "The yeast Prefoldin Bud27," in Prefoldins: the New Chaperones, ed. N. Djouder (Cham: Springer International Publishing), 109118. doi: 10.1007/978-3-030-00737-9_8

Martínez-Fernández, V., and Navarro, F. (2018). Rpb5, a subunit shared by eukaryotic RNA polymerases, cooperates with prefoldin-like Bud27/URI. AIMS Genet. 5, 63-74. doi: 10.3934/genet.2018.1.63

McClellan, A. J., Xia, Y., Deutschbauer, A. M., Davis, R. W., Gerstein, M., and Frydman, J. (2007). Diverse cellular functions of the Hsp90 molecular chaperone uncovered using systems approaches. Cell 131, 121-135. doi: 10. 1016/j.cell.2007.07.036

Minaker, S. W., Filiatrault, M. C., Ben-Aroya, S., Hieter, P., and Stirling, P. C. (2013). Biogenesis of RNA polymerases II and III requires the conserved GPN small GTPases in Saccharomyces cerevisiae. Genetics 193, 853-864. doi: 10.1534/ genetics.112.148726

Minakhin, L., Bhagat, S., Brunning, A., Campbell, E. A., Darst, S. A., Ebright, R. H., et al. (2001). Bacterial RNA polymerase subunit omega and eukaryotic RNA polymerase subunit RPB6 are sequence, structural, and functional homologs and promote RNA polymerase assembly. Proc. Natl. Acad. Sci. U.S.A. 98, 892-897. doi: 10.1073/pnas.98.3.892

Mirón-García, M. C., Garrido-Godino, A. I., García-Molinero, V., HernándezTorres, F., Rodríguez-Navarro, S., and Navarro, F. (2013). The prefoldin bud27 mediates the assembly of the eukaryotic RNA polymerases in an rpb5-dependent manner. PLoS Genet. 9:e1003297. doi: 10.1371/journal.pgen. 1003297

Mirón-García, M. C., Garrido-Godino, A. I., Martínez-Fernández, V., FernándezPévida, A., Cuevas-Bermúdez, A., Martín-Expósito, M., et al. (2014). The yeast prefoldin-like URI-orthologue Bud27 associates with the RSC nucleosome remodeler and modulates transcription. Nucleic Acids Res. 42, 9666-9676. doi: 10.1093/nar/gku685

Moreno-Morcillo, M., Taylor, N. M., Gruene, T., Legrand, P., Rashid, U. J., Ruiz, F. M., et al. (2014). Solving the RNA polymerase I structural puzzle. Acta Crystallogr. Section D Biol. Crystallogr. 70, 2570-2582.

Mosley, A. L., Hunter, G. O., Sardiu, M. E., Smolle, M., Workman, J. L., Florens, L., et al. (2013). Quantitative proteomics demonstrates that the RNA polymerase II subunits Rpb4 and Rpb7 dissociate during transcriptional elongation. Mol. Cell. Proteomics 12, 1530-1538. doi: 10.1074/mcp.m112.024034

Mosley, A. L., Pattenden, S. G., Carey, M., Venkatesh, S., Gilmore, J. M., Florens, L., et al. (2009). Rtr1 is a CTD phosphatase that regulates RNA polymerase II during the transition from serine 5 to serine 2 phosphorylation. Mol. Cell 34, 168-178. doi: 10.1016/j.molcel.2009.02.025

Muñoz, A., Mangano, S., González-García, M. P., Contreras, R., Sauer, M., De Rybel, B., et al. (2017). RIMA-dependent nuclear accumulation of IYO triggers auxin-irreversible cell differentiation in Arabidopsis. Plant Cell 29, 575-588. doi: 10.1105/tpc.16.00791

Niesser, J., Wagner, F. R., Kostrewa, D., Muhlbacher, W., and Cramer, P. (2015). Structure of GPN-Loop GTPase Npa3 and implications for RNA polymerase II assembly. Mol. Cell Biol. 36, 820-831. doi: 10.1128/MCB.01009-15

Nouraini, S., Archambault, J., and Friesen, J. D. (1996). Rpo26p, a subunit common to yeast RNA polymerases, is essential for the assembly of RNA polymerases I and II and for the stability of the largest subunits of these enzymes. Mol. Cell Biol. 16, 5985-5996. doi: 10.1128/mcb.16.11.5985

Pearl, L. H., and Prodromou, C. (2006). Structure and mechanism of the Hsp90 molecular chaperone machinery. Annu. Rev. Biochem. 75, 271-294. doi: 10. 1146/annurev.biochem.75.103004.142738
Peiro-Chova, L., and Estruch, F. (2009). The yeast RNA polymerase II-associated factor Iwr1p is involved in the basal and regulated transcription of specific genes. J. Biol. Chem. 284, 28958-28967. doi: 10.1074/jbc.m109.012153

Reyes-Pardo, H., Barbosa-Camacho, A. A., Pérez-Mejía, A. E., Lara-Chacón, B., Salas-Estrada, L. A., Robledo-Rivera, A. Y., et al. (2012). A nuclear export sequence in GPN-loop GTPase 1, an essential protein for nuclear targeting of RNA polymerase II, is necessary and sufficient for nuclear export. Biochim. Biophys. Acta Mol. Cell Res. 1823, 1756-1766. doi: 10.1016/j.bbamcr.2012.07. 001

Rubbi, L., Labarre-Mariotte, S., Chedin, S., and Thuriaux, P. (1999). Functional characterization of ABC10alpha, an essential polypeptide shared by all three forms of eukaryotic DNA-dependent RNA polymerases. J. Biol. Chem. 274, 31485-31492. doi: 10.1074/jbc.274.44.31485

Rutter, J., Probst, B. L., and McKnight, S. L. (2002). Coordinate regulation of sugar flux and translation by PAS kinase. Cell 111, 17-28. doi: 10.1016/s00928674(02)00974- 1

Schneider, D. A., and Nomura, M. (2004). RNA polymerase I remains intact without subunit exchange through multiple rounds of transcription in Saccharomyces cerevisiae. Proc. Natl. Acad. Sci. U.S.A. 101, 15112-15117. doi: 10.1073/pnas.0406746101

Schopf, F. H., Biebl, M. M., and Buchner, J. (2017). The HSP90 chaperone machinery. Nat. Rev. Mol. Cell Biol. 18, 345-360. doi: 10.1038/nrm.2017.20

Smith-Kinnaman, W. R., Berna, M. J., Hunter, G. O., True, J. D., Hsu, P., Cabello, G. I., et al. (2014). The interactome of the atypical phosphatase Rtr1 in Saccharomyces cerevisiae. Mol. Biosyst. 10, 1730-1741. doi: 10.1039/ c4mb00109e

Staresincic, L., Walker, J., Dirac-Svejstrup, A. B., Mitter, R., and Svejstrup, J. Q. (2011). GTP-dependent binding and nuclear transport of RNA polymerase II by Npa3 protein. J. Biol. Chem. 286, 35553-35561. doi: 10.1074/jbc.m111.286161

Taipale, M., Jarosz, D. F., and Lindquist, S. (2010). HSP90 at the hub of protein homeostasis: emerging mechanistic insights. Nat. Rev. Mol. Cell Biol. 11, 515528. doi: $10.1038 / \mathrm{nrm} 2918$

Uetz, P., Giot, L., Cagney, G., Mansfield, T. A., Judson, R. S., Knight, J. R., et al. (2000). A comprehensive analysis of protein-protein interactions in Saccharomyces cerevisiae. Nature 403, 623-627.

Vernekar, D. V., and Bhargava, P. (2015). Yeast Bud27 modulates the biogenesis of Rpc128 and Rpc160 subunits and the assembly of RNA polymerase III. Biochim. Biophys. Acta Gene Regul. Mech. 1849, 1340-1353. doi: 10.1016/j.bbagrm.2015. 09.010

Victorino, J. F., Fox, M. J., Smith-Kinnaman, W. R., Justice, S. A. P., Burriss, K. H., Boyd, A. K., et al. (2020). RNA Polymerase II CTD phosphatase Rtr1 fine-tunes transcription termination. PLoS Genet. 16:e1008317. doi: 10.1371/journal.pgen. 1008317

Villanyi, Z., Ribaud, V., Kassem, S., Panasenko, O. O., Pahi, Z., Gupta, I., et al. (2014). The Not5 subunit of the ccr4-not complex connects transcription and translation. PLoS Genet. 10:e1004569. doi: 10.1371/journal.pgen.1004569

Wandinger, S. K., Richter, K., and Buchner, J. (2008). The Hsp90 chaperone machinery. J. Biol. Chem. 283, 18473-18477. doi: 10.1074/jbc.r8000 07200

Wani, S., Hirose, Y., and Ohkuma, Y. (2014). Human RNA polymerase IIassociated protein 2 (RPAP2) interacts directly with the RNA polymerase II subunit Rpb6 and participates in pre-mRNA $3^{\prime}$-end formation. Drug Discover. Ther. 8, 255-261. doi: 10.5582/ddt.2014.01044

Werner, F. (2007). Structure and function of archaeal RNA polymerases. Mol. Microbiol. 65, 1395-1404. doi: 10.1111/j.1365-2958.2007.05876.x

Werner, F., and Grohmann, D. (2011). Evolution of multisubunit RNA polymerases in the three domains of life. Nat. Rev. Microbiol. 9, 85-98. doi: 10.1038/nrmicro2507

Werner, M., Thuriaux, P., and Soutourina, J. (2009). Structure-function analysis of RNA polymerases I and III. Curr. Opin. Struct. Biol. 19, 740-745. doi: 10.1016/j.sbi.2009.10.005

Wierzbicki, A. T., Haag, J. R., and Pikaard, C. S. (2008). Noncoding transcription by RNA polymerase Pol IVb/Pol V mediates transcriptional silencing of overlapping and adjacent genes. Cell 135, 635-648. doi: 10.1016/j.cell.2008.09. 035

Wild, T., and Cramer, P. (2012). Biogenesis of multisubunit RNA polymerases. Trends Biochem. Sci. 37, 99-105. doi: 10.1016/j.tibs.2011.12.001 
Zeng, F., Hua, Y., Liu, X., Liu, S., Lao, K., Zhang, Z., et al. (2018). Gpn2 and Rba50 directly participate in the assembly of the Rpb3 subcomplex in the biogenesis of RNA polymerase II. Mol. Cell. Biol. 38:e00091-18.

Zhang, G., Campbell, E. A., Minakhin, L., Richter, C., Severinov, K., and Darst, S. A. (1999). Crystal structure of Thermus aquaticus core RNA polymerase at 3.3 A resolution [see comments]. Cell 98, 811-824. doi: 10.1016/s0092-8674(00) 81515-9

Zhao, R., Davey, M., Hsu, Y. C., Kaplanek, P., Tong, A., Parsons, A. B., et al. (2005). Navigating the chaperone network: an integrative map of physical and genetic interactions mediated by the hsp90 chaperone. Cell 120, 715-727. doi: 10.1016/j.cell.2004.12.024
Conflict of Interest: The authors declare that the research was conducted in the absence of any commercial or financial relationships that could be construed as a potential conflict of interest.

Copyright (c) 2021 Garrido-Godino, Gutiérrez-Santiago and Navarro. This is an open-access article distributed under the terms of the Creative Commons Attribution License (CC BY). The use, distribution or reproduction in other forums is permitted, provided the original author(s) and the copyright owner(s) are credited and that the original publication in this journal is cited, in accordance with accepted academic practice. No use, distribution or reproduction is permitted which does not comply with these terms. 